\title{
Structural and optical properties of germanium nanoparticles
}

\author{
Chung-Wei Lin, Shin-Yen Lin, and Si-Chen Lee ${ }^{\text {a) }}$ \\ Department of Electrical Engineering and Graduate Institute of Electronic Engineering, National Taiwan \\ University, Taipei, Taiwan, Republic of China \\ Chih-Ta Chia \\ Department of Physics, National Normal Taiwan University, Taipei, Taiwan, Republic of China
}

(Received 26 June 2001; accepted for publication 24 October 2001)

\begin{abstract}
Germanium nanoparticles with spherical shape have been prepared by the thermal evaporation method. The shape and structure of these particles have been studied. Transmission electron microscopy images show that a change of Ge structure from amorphous to crystalline occurs at a growth pressure of 0.4 Torr and is attributed to the change of lattice constant. When the growth pressure becomes larger than 0.4 Torr, a fractallike tree structure made of Ge nanoparticles is observed. As the pressure increases further, the dot shape becomes clearer and many dots overlap. From the Raman spectra, it is found that the diamond structure is dominant for Ge dots. (c) 2002 American Institute of Physics. [DOI: 10.1063/1.1428102]
\end{abstract}

\section{INTRODUCTION}

In recent years, $\mathrm{Si}$ and $\mathrm{Ge}$ nanoparticles have attracted much attention because of their possible integration with traditional Si transistors. Nanoparticles are zero dimensional materials, so their electronic structure may transform from indirect band gap to direct band gap which is able to emit light easily. Their band gap also increases due to the quantum confinement effect. The integration of $\mathrm{Si} / \mathrm{Ge}$ optoelectronic device with a Si integrated circuit opens the possibility for an optical link between chips in next generation ultralarge scaled integrated circuits. There are many methods to prepare nanoparticles such as chemical precipitation, ${ }^{1}$ thermal evaporation, ${ }^{2-4}$ chemical vapor deposition, ${ }^{5,6}$ laser ablation, ${ }^{7}$ molecular beam epitaxy, ${ }^{8}$ etc. The thermal evaporation method was chosen in this article to synthesize $\mathrm{Ge}$ nanoparticles because of its simple process. The dot size can be controlled easily by adjusting the gas pressure.

\section{EXPERIMENTS}

The substrate was 7059 glass and was cleaned in the following steps: dipped in $\mathrm{H}_{2} \mathrm{SO}_{4}: \mathrm{H}_{2} \mathrm{O}_{2}$ (3:1) for $30 \mathrm{~min}$, rinsed in de-ionized water for $10 \mathrm{~min}$, then blown dry with $\mathrm{N}_{2}$ gas in order to wash away organic matter. The Ge powders were placed in the tantalum ( $\mathrm{Ta}$ ) boat and the temperature was controlled by adjusting the electric current through the boat. The distance between the boats and the substrate was $13 \mathrm{~cm}$. The air in the chamber was pumped out to about $10^{-4}$ Torr and the chamber was purged with Ar gas three times to get rid of residual water vapor and oxygen. Then the chamber was filled with Ar to the desired pressure and the substrate was cooled down by the cold trap filled with liquid nitrogen. After the boat temperature was raised to the desired one, the shutter between the source and substrate was opened and the dots were deposited on the substrate. Here, the boat

a) Author to whom correspondence should be addressed; electronic mail: sclee@cc.ee.ntu.edu.tw temperatures was set to $1400{ }^{\circ} \mathrm{C}$ and the growth time was 1 min. Finally, the specimens were warmed to room temperature and taken out of the chamber.

\section{RESULTS AND DISCUSSION}

The transmission electron microscopy (TEM) images of Ge nanoparticles grown at 0.2, 0.4 and 1 Torr are shown in Fig. 1. Some special shapes of nanoparticles are seen in these figures. In Fig. 1(a), it is amazing that some nanowires are seen, the film appears to be paste shaped Ge and it is hard to recognize any independent dot in the image. According to Gole et al. ${ }^{9}$ the nanowires can be made by thermal evaporation under about $1000{ }^{\circ} \mathrm{C}$ chamber temperature and about 12 $\mathrm{h}$ growth time. The reason why they are found here is unknown. But the nanowires can hardly be found again in the same growth condition. It may be caused by the fact that the amount of nanowires is too small. It can be seen that some particles seem to exist in Fig. 1(b). This phenomenon will be discussed with the transmission electron diffraction (TED) patterns later. In Fig. 1(c), the particle shape becomes very clear and many particles overlap each other. It is found that the Ge particles seem to form a fiberlike fractal structure. This may be due to the diffusion limited aggregation process, that is, when the particles land on the substrate, they stick to the first particle they touch. Any protrusion in the first few layers tends to capture more particles and grow like a fiber. ${ }^{4}$ The average particle size and the standard deviation as a function of growth pressure of Ge particles are shown in Fig. 2. It is clear that while the growth pressure becomes larger, the particle size grows bigger. This is because when the pressure is larger, the collision of Ge particles is more frequent at higher pressure. Figure 2(b) displays the standard deviation of the Ge particle size, which is a linear function of the growth pressure. Yatsuya et al. ${ }^{10}$ had already given a good explanation of this. Typical smoke on top of the evaporation source consists of the inner zone, inner front, outer zone, and outer front. Smoke particles often showed clear-cut habits in 


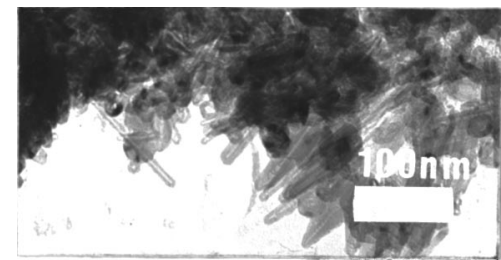

(a)

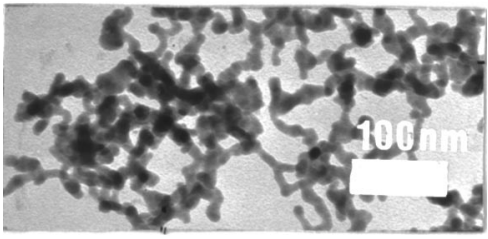

(b)

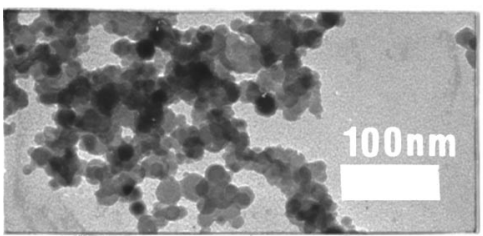

(C)

FIG. 1. The TEM images of Ge nanoparticles grown at: (a) 0.2, (b) 0.4, and (c) 1 Torr.

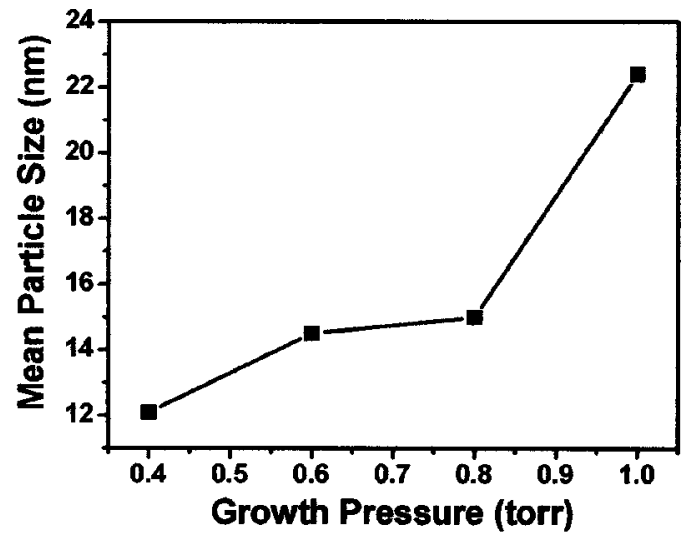

(a)

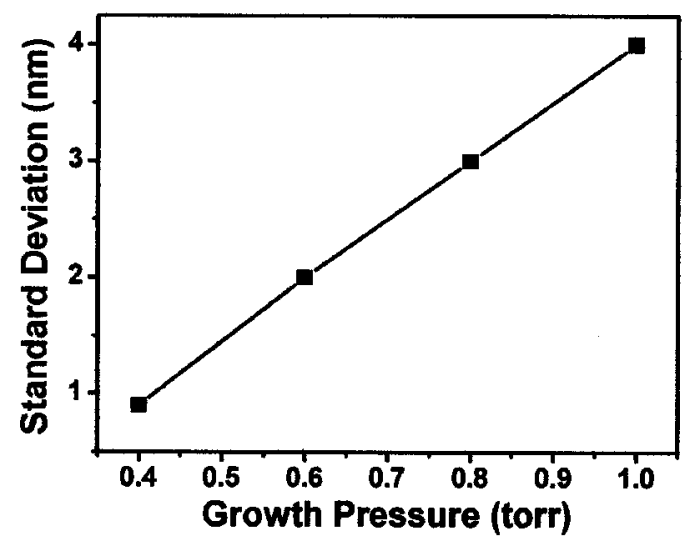

(b)

FIG. 2. (a) The mean particle size and (b) the standard deviation as a function of the growth pressure of Ge dots.

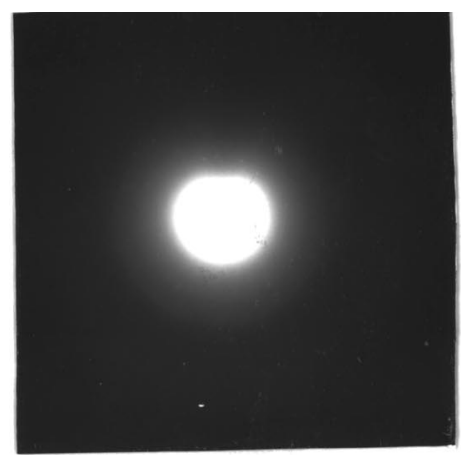

(a)

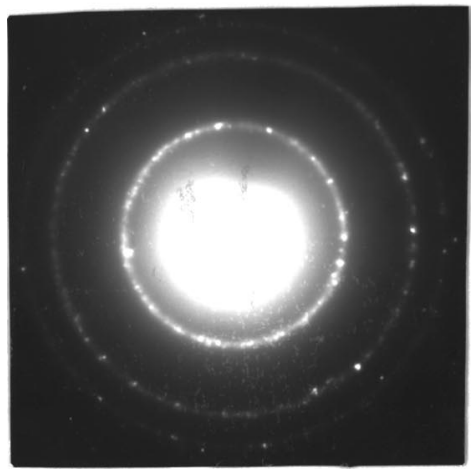

(b)

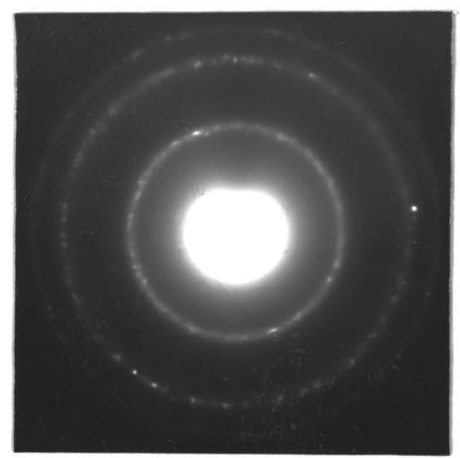

(C)

FIG. 3. The TED patterns of Ge nanoparticles grown at: (a) 0.2 , (b) 0.4 , and (c) 1 Torr.

the inner zone, and the particles collected in the inner zone are nearly uniform in size. When the pressure is high, the inner zone becomes small, so the standard deviation becomes larger.

Figures 3(a), 3(b) and 3(c) display the TED patterns of germanium particles grown at $0.2,0.4$, and 1 Torr, respectively. It is clear that while the growth pressure is higher than 0.4 Torr, the TED patterns change from amorphous to polycrystalline germanium, i.e., (220) and (311) rings appear as evidenced from Figs. 3(a)-3(c). The nanoparticles have a diamond structure. The (220) ring is chosen to compare the lattice constant of Ge particles prepared at different pressure because it is large and easy to measure. The extracted lattice constant of Ge particles grown at 0.4 Torr is about $5.54 \AA$ and that of Ge particles grown at 1 Torr is about $5.70 \AA$. It means that the lattice constant of Ge nanoparticles shrinks slightly with shrinking particle size. However, if the lattice constant shrinks too much, the diamond structure will col- 


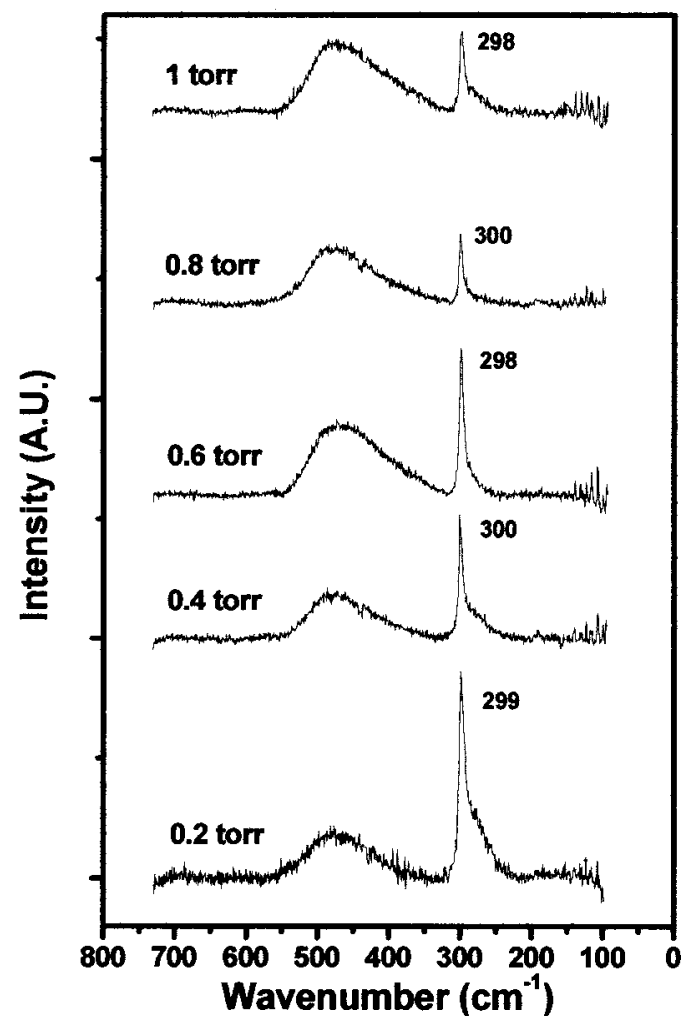

FIG. 4. The Raman spectra of Ge nanoparticles grown at 0.2, 0.4, 0.6, 0.8, and 1 Torr.

lapse and result in the paste shape. This may be caused by the rapid oxidation of Ge particles when they are taken out from the chamber. The film immediately turns from a deep brown to a brown color, indicating that a fierce chemical reaction occurs. This rapid oxidation may compress the lattice constant and if the dot size is not large enough to sustain the force, the diamond structure will collapse and no ring is found in the TED pattern. The oxide thickness on SiGe nanoparticles had been found by Liao et al. ${ }^{4}$ to be $2.5 \mathrm{~nm}$. For Ge nanoparticles the oxide thickness should be thicker, i.e., $\sim 3$ $\mathrm{nm}$, because Ge oxidized easier than Si. From Fig. 2(a), the critical diameter of Ge nanoparticles is found to be about 12 $\mathrm{nm}$. This includes the surface oxide thickness. Therefore the real critical size of Ge nanoparticles may be smaller than 6 $\mathrm{nm}$. For nanoparticles with smaller size the diamond structure becomes unstable and a transition from crystalline to amorphous structure will occur.

Figure 4 shows the Raman spectra of Ge nanoparticles grown at different pressure from 0.2 to 1 Torr. Because nanoparticles are grown on 7059 glasses, the broad peak around $480 \mathrm{~cm}^{-1}$ comes from the substrate. The two main Raman peaks of $\mathrm{Ge}$ are located at 300 and $270 \mathrm{~cm}^{-1}$, which correspond to phonons from diamond structure $\mathrm{Ge}$ and tetragonal structure Ge, respectively. ${ }^{11}$ It can be seen that the peak located at $300 \mathrm{~cm}^{-1}$ is sharp and the peak located at $270 \mathrm{~cm}^{-1}$ seems to be weak and broad, so only the diamond structure can be detected in the TED patterns. But the result of the TED pattern is different from that of the Raman spectrum of Ge dots grown at 0.2 Torr. From the Raman spectrum, the diamond structure is formed but no ring is seen in the TED

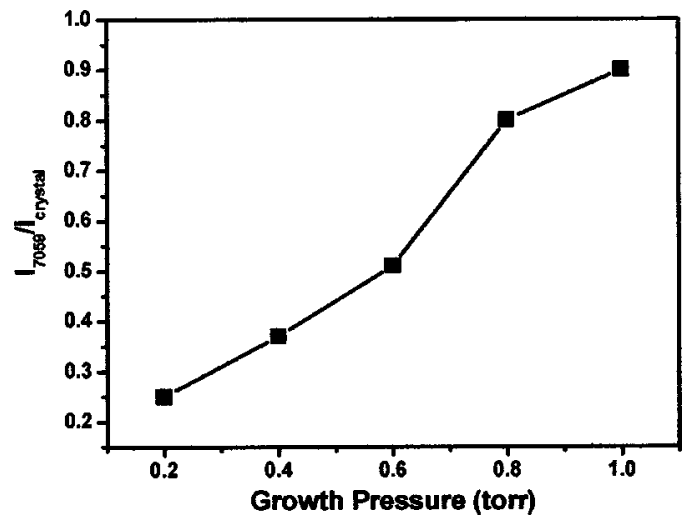

FIG. 5. The Raman signal ratio between the substrate and crystalline parts.

pattern. This may be caused by the different spot size and penetration depth of the electron beam and the Ar laser. The spot size and penetration depth of the Ar laser are larger, so many nanowires may be detected and the Raman peak located at $300 \mathrm{~cm}^{-1}$ appears in the spectrum. Another interesting phenomenon is the peak intensity ratio of $480-300$ $\mathrm{cm}^{-1}$. Figure 5 displays the Raman peak ratio between the glass substrate and crystalline Ge film. It is clear that while the growth pressure is larger, the ratio becomes larger. But it is known that the diamond structure is dominant with larger growth pressure, so it may be caused by the greater scattering of light from the sample. That is, the mean particle size and their standard deviation will be larger at higher growth pressure, so the sample surface becomes rougher, which enhances the scattering of the light. Figure 6 shows the Raman signal ratio between the tetragonal and diamond parts. It is calculated by dividing the intensity of the Raman peak located at $270 \mathrm{~cm}^{-1}$ by the peak located at $300 \mathrm{~cm}^{-1}$. From this figure, it can be found that the ratio becomes small and the tetragonal peak almost disappears at 100 Torr growth pressure, so only the diffraction rings of the diamond structure can be seen and they are clearer because the ratio of the diamond structure becomes larger.

\section{CONCLUSIONS}

The Ge nanoparticles have a diamond structure, where their average particle size and the standard deviation become

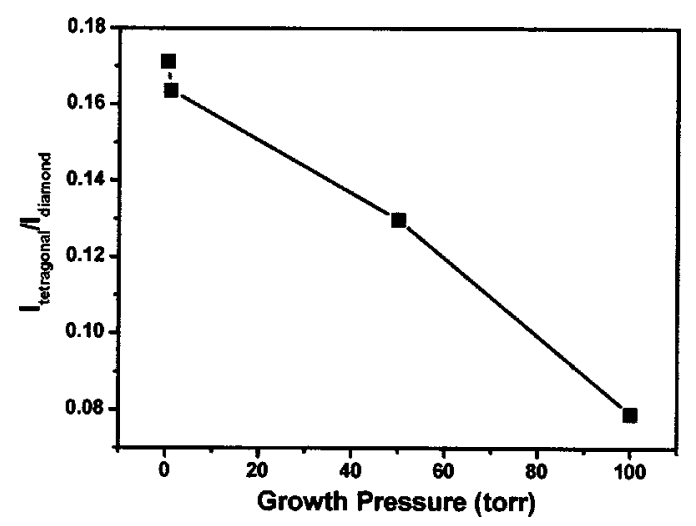

FIG. 6. The Raman signal ratio between the tetragonal and diamond parts. 
larger as the growth pressure becomes larger. If the growth pressure is lower than 0.4 Torr, these crystalline particles will disappear, and instead an amorphous film is produced. The critical nanoparticle size is about 4-6 nm excluding the surface oxide thickness. From the Raman spectra, it is shown that the diamond rather than tetragonal structure is dominant for Ge nanoparticles.

\section{ACKNOWLEDGMENT}

This work is supported by National Science Council of the Republic of China under Contract No. NSC90-2215E002-010.
${ }^{1}$ Z. X. Tang, C. M. Sorenson, K. J. Klabunde, and G. C. Hadjipanayis, J. Colloid Interface Sci. 146, 38 (1991).

${ }^{2}$ Y. Kawamura, M. Takagi, and M. Akai, Mater. Sci. Eng. 98, 449 (1988).

${ }^{3}$ R. W. Siegal, Mater. Sci. Eng. A 168, 189 (1993).

${ }^{4}$ Y. C. Liao, S.-Y. Lin, S.-C. Lee, and C.-T. Chia, Appl. Phys. Lett. 77, 4328 (2000).

${ }^{5}$ Y. He, H. Liu, N. Yu, and X. M. Yu, Nanostruct. Mater. 7, 769 (1996).

${ }^{6}$ Y. He, C. Yin, G. Cheng, L. Wang, and X. Liu, J. Appl. Phys. 75, 797 (1994).

${ }^{7}$ N. L. Mandich, V. E. Bondybey, and W. D. Reents, J. Chem. Phys. 86, 4245 (1987).

${ }^{8}$ E. Gaffet and M. Harmelin, J. Less-Common Met. 157, 201 (1990).

${ }^{9}$ J. L. Gole, J. D. Stout, W. L. Rauch, and Z. L. Wang, Appl. Phys. Lett. 76, 2346 (2000).

${ }^{10}$ S. Yatsuya, S. Kasukabe, and R. Uyeda, Jpn. J. Appl. Phys. 12, 1675 (1973).

${ }^{11}$ M. Wakaki, M. Iwase, Y. Show, K. Koyama, S. Sato, S. Nozaki, and H. Morisaki, Physica B 219\&220, 535 (1996). 